\title{
Remote temperature monitoring device using a multiple patients-coordinator set design approach
}

\author{
Cheng S Chin ${ }^{1 *}$, William Atmodihardjo ${ }^{2}$, Lok W Woo ${ }^{1}$ and Ehsan Mesbahi ${ }^{1}$
}

\begin{abstract}
This paper aims to develop a prototype for a web-based wireless remote temperature monitoring device for patients. This device uses a patient and coordinator set design approach involving the measurement, transmission, receipt and recording of patients' temperatures via the MiWi wireless network. The results of experimental tests on the proposed system indicated a wider distance coverage and reasonable temperature resolution and standard deviation. The system could display the temperature and patient information remotely via a graphical-user interface as shown in the tests on three healthy participants. By continuously monitoring participants' temperatures, this device will likely improve the quality of the health care of the patients in normal ward as less human workload is involved.
\end{abstract}

Keywords: Temperature; Wireless; Web-based; Remote; Monitoring; Patient set; Coordinator set

\section{Background}

During the severe acute respiratory syndrome (SARS) outbreak in 2003, hospitals became treatment centres in most countries. Because a patient's core body temperature is one vital parameter for monitoring the progress of the patient's health, it is often measured manually at a frequency ranging from once every few hours to once a day [1]. However, such manual measurement of the temperature of patients requires the efforts of many staff members. In addition, when the patients suffer from conditions that result in abrupt changes of the core body temperature, e.g., due to infection at a surgical site after surgery, the staff on duty will not know such a temperature change occurred until the next temperature measurement. Such a delay may lead to patients being unnoticed while their health conditions worsen, which is dangerous because a difference of 1.5 degrees Celsius can result in adverse outcomes [2]. Furthermore, there is always a need to have a monitoring system to improve the quality of health care [3], such as temperature monitoring of elderly and challenged persons using a wireless remote temperature monitoring system.

Body temperature can be used to monitor the pain level of a patient following an operation [4] or after

\footnotetext{
* Correspondence: cheng.chin@ncl.ac.uk

${ }^{1}$ Faculty of Science Agriculture and Engineering, University of Newcastle upon Tyne, Newcastle upon Tyne NE1 7RU, United Kingdom

Full list of author information is available at the end of the article
}

shoulder endoprosthesis [5]. In some cases, the tissue transient temperature was monitored during microwave liver ablation [6] for the treatment of liver metastases. Instead of using a temperature sensor, pulse-echo ultrasound [7] was used to visualize changes in the temperature of the patient's body. In addition, a non-contact temperature-measuring device, such as a thermal imaging camera [8], was successfully used to detect human body temperature during the SARS outbreak. However, it can be quite expensive to equip each patient room with a thermal imaging camera. In addition, there are a few wireless temperature-monitoring systems (e.g., CADIT $^{\mathrm{Tm}}$, Primex ${ }^{\mathrm{Tm}}$, and TempTrak ${ }^{\mathrm{Tm}}$ ) on the market that are used to monitor and store a patient's temperature for medical research by using body sensor networks [9]. Most of these systems consist of an electronic module and a temperature-sensing device. The systems include a stand-alone electronic module with a display screen that allows the temperature sensor data to be transmitted over a secure wireless network.

However, these systems can be difficult to reconfigure to suit the current database system used in the hospital. In addition, the current systems using short message service (SMS)-based telemedicine [10] systems with hardware equipment were developed to monitor the mobility of patients. However, proper hardware and software to manage the messages and the patient's temperature for display on mobile phones are not widely available. 
Hence, a medical device to continuously measure the body temperature of patients using a wireless temperature sensor $[4,11,12]$ is required. With such a wireless temperature sensor system, nurses will no longer have to manually measure the temperature of patients, which will free their time for other tasks and also reduce the risk associated with coming into contact with patients with contagious diseases, such as SARS. The readings will be transmitted wirelessly to the central nurse station, where they can be monitored by the staff-on-duty. In addition, the current and past history of the body temperature measurements can be stored in an online database, which allows the medical staff to access the database when they are not in the hospital.

To the best of our knowledge, a MiWi wireless (besides using the Zigbee[11]) temperature-monitoring system using a patient and coordinator set design that provides remote internet access to the temperature database has not been reported in any publication. The objective is therefore to develop and implement a prototype temperature-monitoring system for patients using a MiWi wireless remote connection to the nurse's station for frequent real-time monitoring. The temperature monitoring system was designed based on a proposed patient and coordinator set design approach. The proposed temperature-monitoring system for use in normal ward will likely to improve the quality of the health care of the patients as the nursing workload is reduced. In this paper, the discussion on medical regulations and policy will not be included.

\section{Method}

\section{Design concepts of the wireless remote temperature- monitoring system}

The core body temperature of a human is one of the vital statistics used to determine individual health. Normal human body temperature (normothermia) is approximately $37^{\circ} \mathrm{C}$, with variations depending on factors such as age and geological factor [13]. When the body fails to regulate itself by dissipating heat, it increases the core body temperature above the normal condition. This condition is called hyperthermia. The highest recorded surviving core body temperature is $45^{\circ} \mathrm{C}$ [14]. Generally, there are four locations [15] that can be used to measure human core body temperature, rectal (anus), oral (mouth), tympanic (ear) and axillary (armpit), with the rectal measurement considered to be the most accurate in measuring the core body temperature. Rectal measurement of body temperature is the preferred method to perform a core body temperature measurement. In order to track a patient's core body temperature wirelessly (assuming the patient is not moving), the axillary measurement was chosen for the initial trial. The proposed measurement system must be able to measure within the temperature deviation $[16,17]$ of $0.3-0.5^{\circ} \mathrm{C}$ and detect changes in the human body temperature (at minimum resolution of $0.1^{\circ} \mathrm{C}$ ).

\section{MiWi wireless network}

The originality of the paper is to develop and test a prototype temperature-monitoring system for patients using the MiWi wireless remote connection to the nurse's station for frequent real-time monitoring. As this project aims to be implemented in a hospital environment, when choosing the appropriate wireless technology it should be noted that the chosen technology should be the best choice in terms of cost, connectivity range, speed, etc. for the chosen environment. Table 1 below depicts the comparison between ZigBee, Bluetooth and Wi-Fi.

It is concluded from the table that ZigBee is the most fitting technology for this project. The main reason for this decision is because ZigBee requires very low power consumption by staying on sleep mode most of the time while still having the same range potential as Bluetooth and Wi-Fi. Another advantage of using ZigBee comes from having more possible topology which is star that allows 1 FFD (Full Function Device) unit to control multiple RFD (Reduced Function Device) units and mesh which allows the system to be more resistant to RFD unit breaking down as can be seen on Figure 1. While

Table 1 Wireless technologies comparison

\begin{tabular}{llll}
\hline Descriptions & ZigBee & Bluetooth & Wi-Fi \\
\hline Applications & Control and monitoring, sensor networks & Wireless connection between devices & Wireless LAN, internet access \\
\hline Data rate & 20,40, and 250 kbps & $1 \mathrm{Mbps}$ & 11 and $55 \mathrm{Mbps}$ \\
\hline Frequency band & $868 \mathrm{MHz}, 900-928 \mathrm{MHz}$, and 2.4GHz & $2.4 \mathrm{GHz}$ & 2.4 and $5 \mathrm{GHz}$ \\
\hline Range (meters) & $10-100$ & 10 & $50-100$ \\
\hline Nodes per network & 65,000 & 7 & 30 \\
\hline Memory & $32-60 \mathrm{~Kb}$ & $100 \mathrm{~Kb}$ & $100 \mathrm{~Kb}$ \\
\hline Topology & Star, tree, and mesh & Tree & Tree \\
\hline Complexity & Low & High & High \\
\hline Power consumption & Very low & Medium & High \\
\hline Standby current & $3 \mu \mathrm{A}$ & $200 \mu \mathrm{A}$ & $20 \mu \mathrm{A}$ \\
\hline
\end{tabular}




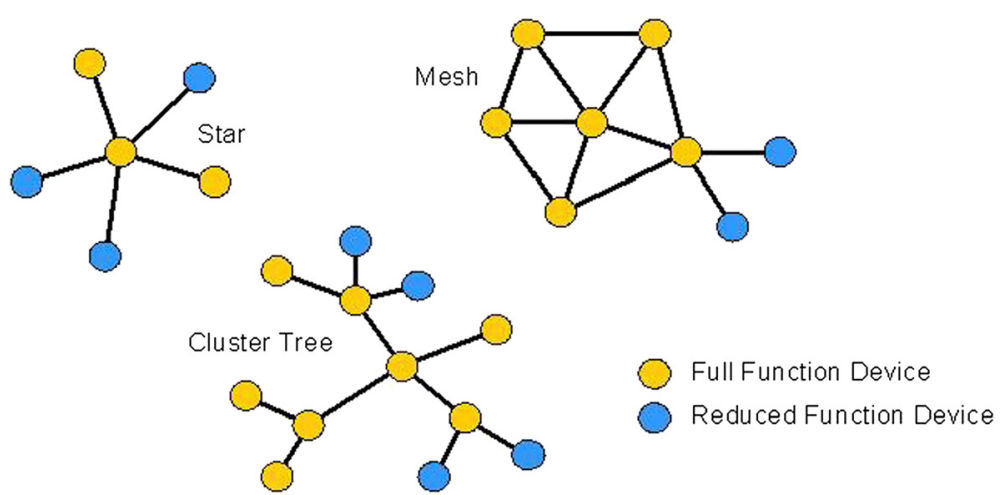

Figure 1 Different ZigBee topologies.

ZigBee has lower data rate than the other two, it is sufficient for transmitting the temperature data that will be measured in this project.

ZigBee's is mostly used in low power, low data rate application such as home monitoring system due to its benefits compared to other wireless technologies as explained in Table 1. Each device can be configured into two different types: FFD which can communicate with both types and RFD which can only communicate with FFD type. FFD can be configured into a coordinator which is the base station in this project, or router which serves to extend the range of the system. FFD is configured as patient's unit in this project. The ZigBee network itself works in layers as shown in Figure 2 with each having specific task to manage the wireless connection. The PHY (physical) layer manages data transmission by performing quality checking on both channel frequency and in/outgoing signals and also managing the RF transceiver module. Some of the specific tasks it needs to do are energy detection, link quality indication, clear channel assessment and channel selection. The MAC (Media Access Control) layer manages the access of data into the frequency channel and control the network beaconing. It also assigns time slots for the transmitted data and manages data security. The NWK (network) layer manages the whole network connectivity by doing tasks such as ensuring the MAC layer is used correctly and identifying whether a neighbouring device belongs to the system or not. The NWK layer is also responsible for creating a suitable interface for highest APL (application) layer in which the users can interact with the ZigBee application and interface.

Unfortunately, the ZigBee protocol stack is too large for most commonly used MCUs' memory to handle. Therefore MiWi protocol stack will be used instead. MiWi wireless transceiver (MRF24J40MA) used in the project support P2P protocol. There is no significant different from other P2P protocols. But when compared with the ZigBee protocol stack, ZigBee protocol is too large for most commonly used MCUs' memory to handle. The reason is that MiWi protocol stack only uses the MAC and PHY layers which reduce the code used. Another merits using MiWi P2P are the protocol is proprietary and it is not interoperable. In addition, MiWi P2P has also a small footprint and a very low overhead as compared with ZigBee protocol. However, the demerits of MiWi P2P are the small network size and only allow small number of hops.

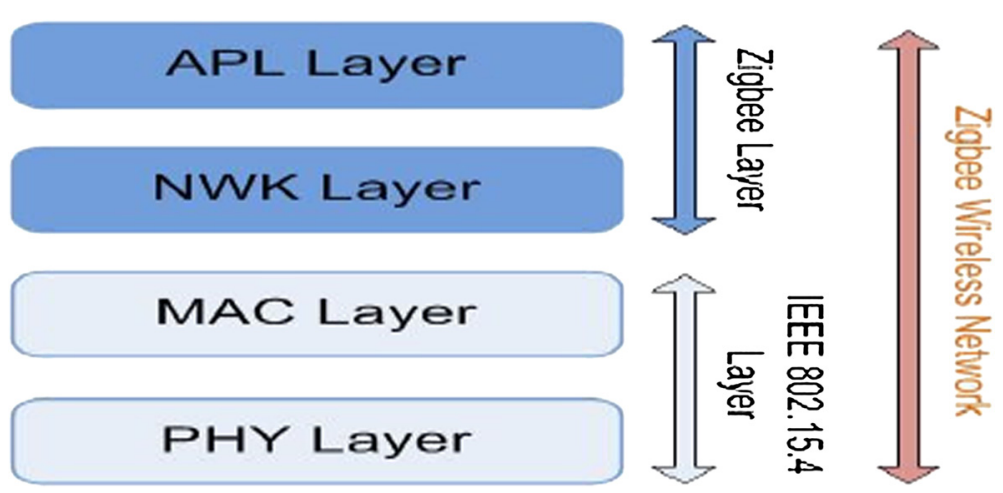

Figure 2 ZigBee wireless network layers. 
When compared to grid networks which provide many servers to moderate-sized communities and emphasize the integration of resources to deliver in an environment of at least limited trust, P2P deal with many more participants but offer limited and specialized servers, have been less concerned with qualities of service, and limited assumptions about trust. But as system scale increases in the grid networks, it may face and address problems relating to autonomic configuration and management. On the other hand, the P2P developed rapidly around sharing and are now seeking to expand to more sophisticated applications and management.

\section{Multiple patients-coordinator set design approach}

To accomplish the axillary temperature measurement, the wireless remote temperature monitoring system design (see Figure 3) includes a MiWi wireless transceiver (MRF24J40MA), a PIC microprocessor (PIC18LF4620)/ $\mathrm{MCU}$, a temperature sensing circuit, a patient set (on the patients) and a coordinator set (at the nurse's station) for the data coordination between the patients and the central nurse station. The temperature sensor produced an output voltage (and power consumption of $0.24 \mathrm{~mW}$ ) with a magnitude equal to the body temperature. The analogue-to-digital convertor (ADC) had 13 analogue channels and a 10-bit resolution in the microprocessor to convert the output voltage into digital values. The patient's transceiver transmitted that digital value via the MiWi wireless protocol stack to the coordinator's transceiver. The coordinator MCU sent the temperature reading to the computer through RS232 that records and stored the temperature measurements in the database. The proposed wireless temperature-monitoring device could support multiple patients when they were connected to the same wireless network.

There are a few wireless and wired temperature sensors available to monitor the temperature of patients. The temperature resolution is approximately $+/-0.2^{\circ} \mathrm{C}$ for the range $25-60^{\circ} \mathrm{C}$. However, a $1.5^{\circ} \mathrm{C}$ loss [2] in body temperature, e.g., due to surgical site infection after surgery, may lead to adverse outcomes. Hence, a resolution of $+/-0.1^{\circ} \mathrm{C}$ is required. Because the highest recorded surviving core body temperature is $45^{\circ} \mathrm{C}$, the temperature range required for the temperature sensor is reduced. For initial prototyping, a non-medical-grade LM35CAZ temperature sensor with a maximum range of $45^{\circ} \mathrm{C}$ was used. The temperature sensor used draws only $60 \mu \mathrm{A}$ from its supply and power consumption is approximately $0.24 \mathrm{~mW}$. The following is the specifications of the LM35CAZ temperature sensor.

- Calibrated Directly in ${ }^{\circ}$ Celsius (Centigrade)

- Linear $+10 \mathrm{mV} /{ }^{\circ} \mathrm{C}$ Scale Factor

- $0.5^{\circ} \mathrm{C}$ Ensured Accuracy (at $+25^{\circ} \mathrm{C}$ )

- Rated for Full $-55^{\circ} \mathrm{C}$ to $+150^{\circ} \mathrm{C}$ Range

- Operates from 4 to $30 \mathrm{~V}$

- Less than 60- $\mu \mathrm{A}$ Current Drain

- Low Self-Heating, $0.08^{\circ} \mathrm{C}$ in Still Air

- Low Impedance Output, $0.1 \Omega$ for $1 \mathrm{~mA}$ Load

Thus, the only advantage compared to the medicalgrade sensor is the low power consumption. Nevertheless, the sensor can be replaced with any medical-grade temperature probe (such as a standard stick-on skin probe) with minor changes in the hardware and software.

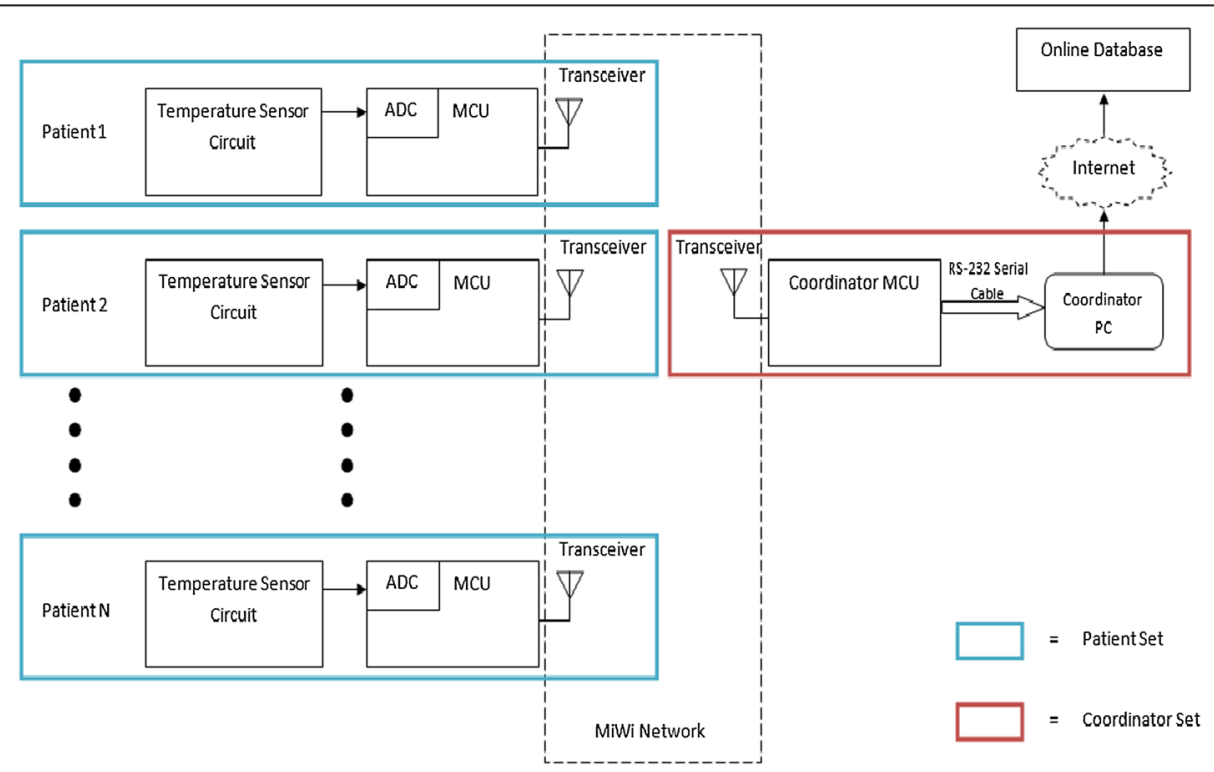

Figure 3 Overall block diagram of the multiple patients-coordinator set design approach. 


\section{Patient set design}

The patient set consists of three main components: temperature sensor circuit, MCU and transceiver. The electronic components were mounted onto a small PCB board and connected to the patient's MCU. As shown in Figure 4, the setup (within the enclosure) was strapped to the patient's arm during temperature monitoring.

Initially, only two patient sets were used. When the patient MCU was turned on, it established a connection with the coordinator set using a wireless protocol stack. For the initial phase of the project, the easy-to-use alternative for simple wireless communication of MiWi (by MicroChip) was used to implement the low-cost and power wireless control networks (with a battery usage of 95-115 $\mu \mathrm{W}$ compared with ZigBee at approximately $30 \mathrm{~mW}$ ).

A MiWi wireless transceiver module (MRF24J40MA2.5GHz IEEE 802.15.4) was mounted onto a carrier board. To allow easy configuration and to monitor packet transactions, a ZENA wireless network analyser (DM183023) and software were used. Figure 5 (top) shows an example of a transmitted message viewed on the ZENA network analyser. The first line indicates the patient node attempting to join a network, while the second line indicates the coordinator recognizing the patient node as a member of the network.

Because the ADC has 10 bits, the output was separated into two registers (two bytes). The 2-byte digital output values were sent to the coordinator set with an additional byte to indicate which patient set sent the data. Sometimes, there may be a disruption or glitch in the MiWi wireless network that prevents the coordinator from receiving the data from the patient. To overcome this issue, a cyclic redundancy check (CRC) of 16 bits (or 2 bytes in length) was used to determine whether the packet was correctly transmitted. For example, if the entire set of bits was correct, the CRC value matched. FCS had the OK bit in the last byte of the data. However, if a single bit was incorrect, the data need to be retransmitted.
The patient set temperature conversion process from the temperature sensor is as follows. The temperature sensor of Patient Set 1 produced an output voltage of $0.365 \mathrm{~V}$ or $36.5^{\circ} \mathrm{C}$. This voltage was amplified 5.12 times to $1.868 \mathrm{~V}$. The ADC converted the voltage into two registers of 1 byte each (conversion to hexadecimal) to obtain 02 46. Subsequently, the MCU added an additional byte to identify the patient set. If Patient Set 1 was used, the extra byte was 01 . Subsequently, the transceiver transmitted the 3 bytes to the coordinator MCU and displayed the temperature on the computer with the GUI.

\section{Coordinator set design}

As shown in Figure 5 (centre), the coordinator set requires a similar MCU and transceiver in the patient set. Each patient set was designed to send a message of 3 bytes to the coordinator MCU. The coordinator's MCU performs a few tasks, as shown in the flow chart. The tasks was to establish a connection with each patient set, send an acknowledgement byte to the patient set after receiving the message, and send the message to the coordinator. The GUI of the coordinator set created a text file containing the temperature reading for a day. The file was stored in the local PC folder with all of the temperature data, the patients' name (with serial or patient number) and the time stamps. The graphical representation of the temperature distribution of the patient is shown in Figure 5. Statistical information, such as the mean and standard deviation for certain time frame, was then computed. The information was displayed on the staff computer near the coordinator patient set.

\section{Patient and coordinator set wireless transmission tests}

The hardware setup in the laboratory consists of the temperature sensor, amplifier circuit, coordinator $\mathrm{MCU}$ and patient MCU, as shown in Figure 4 (right). The output voltage was calibrated and compared with a digital

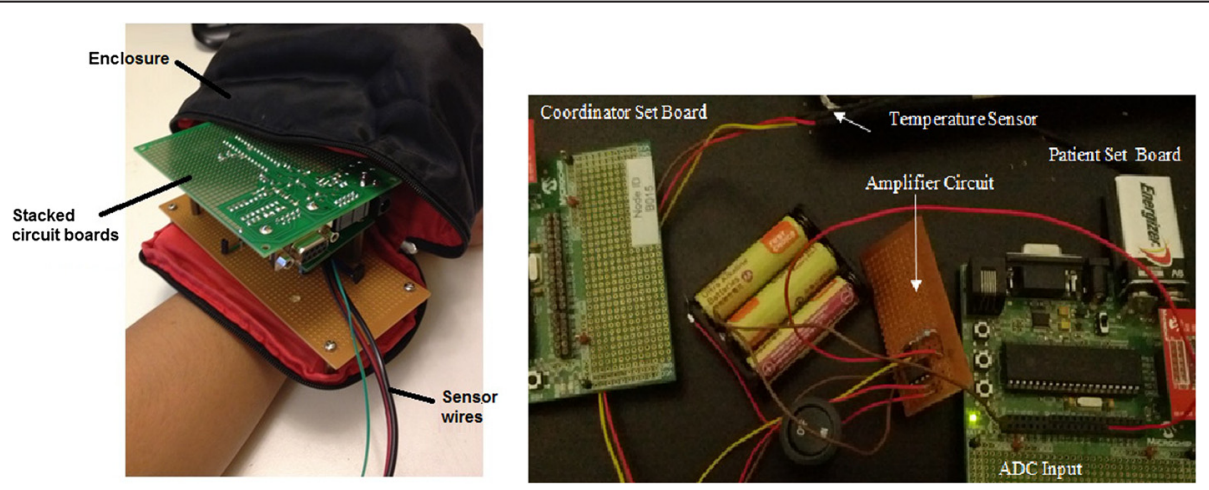

Figure 4 Prototype temperature-monitoring system strapped on patient set 1 (left) and circuit board (right). 


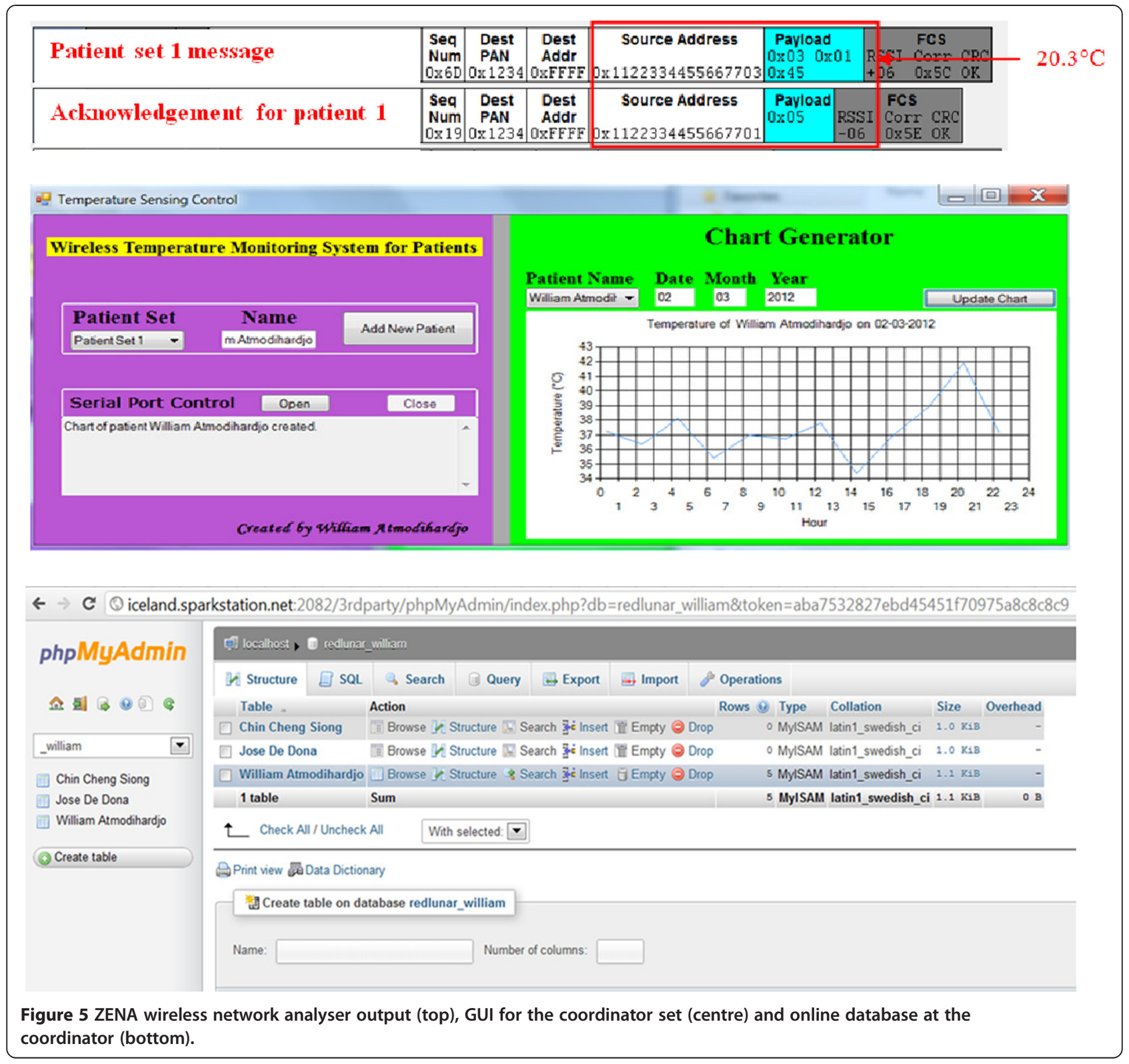

thermometer (i.e. OMRON ${ }^{\mathrm{m}}$ digital thermometer) reading for a few times. It was observed that the sensor output voltage would yield a tolerance level of $\pm 0.2^{\circ} \mathrm{C}$ when compared with the thermometer readings. In addition, the amplifier circuit had an input voltage of $307.4 \mathrm{mV}$ that was amplified 5.12 times to $1.573 \mathrm{~V}$ and displays the result of the ADC process in the ZENA network analyser. The ADC converted the input voltage to the hexadecimal corresponding to $20.3^{\circ} \mathrm{C}$, as shown in Figure 5 (top). The test indicated that the ADC conversion process in the patient's MCU was able to measure the temperature correctly.

As mentioned, the patient MCU sent the 3-byte message to the coordinator MCU. To test this feature, the patient set transmitted the message every few seconds to the coordinator to process the message. The coordinator MCU sent an acknowledgement message until the CRC was shown positive. As shown in Figure 5 (top), an additional patient has the first byte of its message changed to differentiate between different patients. Figure 5 also shows that the coordinator (source address ending with 01 at the payload section) was able to receive messages from multiple patients.

The network coverage range of the MRF24J40MA transceiver was conducted in a school campus to test the network coverage range. The coordinator MCU was placed in a room, while a patient MCU was along the corridor. The coordinator MCU sent a message every few seconds. The signal could be transmitted with a clear line of sight to a distance of approximately $50 \mathrm{~m}$. 
In addition to testing the indoor network coverage, the range of 120 meters provided in the datasheet was validated in a test outdoors at an ambient temperature of $26^{\circ} \mathrm{C}$.

\section{Results}

\section{Actual implementation of the proposed system on patients}

The proposed wireless remote temperature monitoring device was tested on three healthy participants at a classroom (with computers) in the school environment for three different days. We took their temperature when they were sitting during the experiment. The sensor was attached to the body during the measurement only. They took the sensor off when they wanted to relax and lay on foldable beds.

The authors had received the ethics approvals and informed consent from the three participants before the tests were conducted.

The GUI of the coordinator set (for the nurse's station) in Figure 5 (centre) was used. The coordinator set was connected to the coordinator computer via the RS-232 serial cable. Each time the coordinator MCU received a temperature reading message, it sent the message to the coordinator's computer. Upon clicking on the 'Update Chart' button, the GUI read and generated the temperature record chart for that particular patient and date. Once the message was received, the GUI converted the inputs to actual temperature reading.

The user can allocate a patient name tagged with his/ her admission serial number. Once allocated, the patient name with the serial number was applied to the local folder in the database. Figure 5 (bottom) shows the local temperature record of a subject. The temperature record was saved in the text file, and the data were formatted as "Temperature-Time".

In the nurse's station, the two main components used in the implementation of the online database were the
MySQL database and the PHP scripts. An online hosting server called sparkstation.net was used. Each set of patient information includes the temperature, time, and date created by the coordinator. To operate the system properly, the users were trained to retrieve and provide the correct patient information. There was an added feature of using different colours to indicate the patients with high fever; in addition, in the event of a large standard deviation (or equivalent to a sharp slope), an alarm would be triggered.

Statistical information, such as the mean and the standard deviation, for evaluating the data variation was determined. For an initial trial, 20 minutes of recording and sampling at an interval of 2 minutes was conducted to test the proposed system. The subsequent temperature recording was sampled or taken in every hour. The mean of the participant temperature was 36.6 (95\% confidence intervals of 35.9 and 37.3). The standard deviation was 1.0 (95\% confidence intervals of 0.7 and 1.8), and the median was 36.9 (95\% confidence intervals of 36.0 and 37.2).

The wireless remote temperature monitoring system was tested in three different days (named D1, D2 and D3) on three healthy male participants (named P1, P2 and P3) with ages ranging from 28 to 30 years. The interval between each measurement was set to one hour, starting at $1 \mathrm{am}$. The body temperatures of the three participants were recorded and plotted on a time series plot, as shown in Figure 6. The statistical mean temperatures for $\mathrm{P} 1, \mathrm{P} 2$, and $\mathrm{P} 3$ on different days were $36.8^{\circ} \mathrm{C}, 36.7^{\circ} \mathrm{C}$, and $37.8^{\circ} \mathrm{C}$, respectively, and the statistical standard deviations for $\mathrm{P} 1, \mathrm{P} 2$, and $\mathrm{P} 3$ were approximately $0.3^{\circ} \mathrm{C}$. These values were computed using the temperature data collected over 24 hour.

As seen in Figure 6, the wireless remote temperature monitoring system was able to measure the temperatures of the participants from 1st to 24th hour. The highest

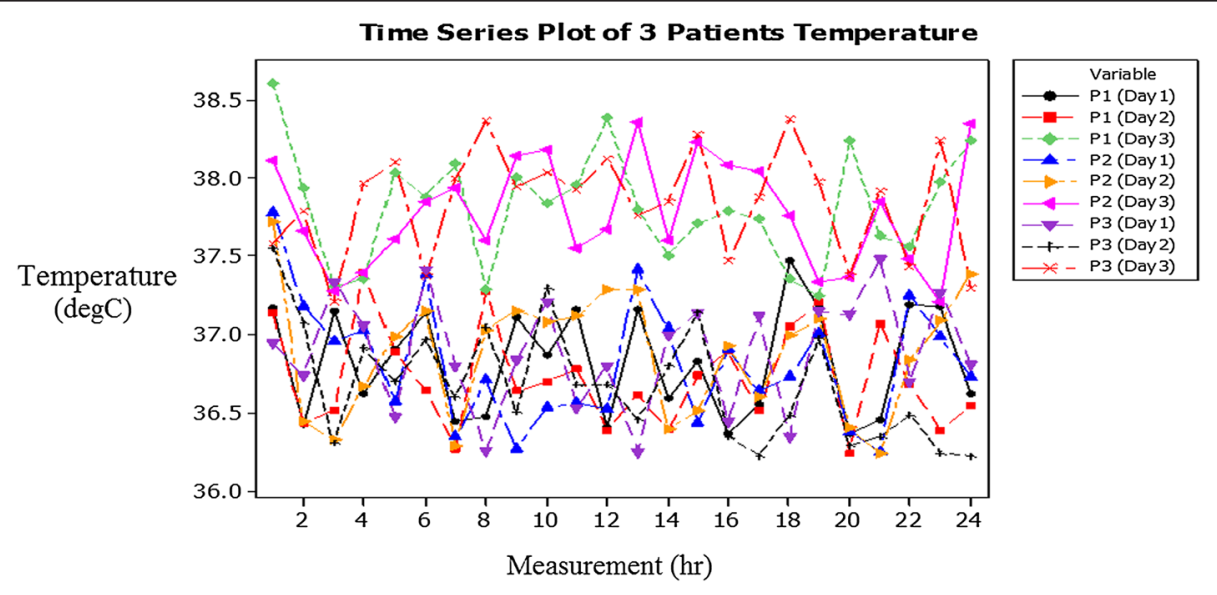

Figure 6 Temperature of three different participants over three days. 
standard deviation was approximately $0.3^{\circ} \mathrm{C}$, which is within the range of 0.3 to $0.5^{\circ} \mathrm{C}[16,17]$ in an enclosed building environment throughout the test period. In summary, the proposed system could measure body temperature within the resolution of 0.3 to $0.5^{\circ} \mathrm{C}$ and exhibit small standard deviation of $0.3^{\circ} \mathrm{C}$.

\section{Discussions}

The temperature readings of the participants who wore the portable temperature monitoring devices were transmitted wirelessly to the main station where they were monitored. The body temperature measurements were stored in an online database, which allowed the user to access the database when they were not with the participants.

The target patients using the device are for those in the normal wards instead of intensive care unit (ICU). Although the monitoring once a day is enough to detect the adverse or other problems because the nurses go around the normal wards to see the patients, the proposed device helps to free their time for other tasks and also reduce the risk associated with coming into contact with patients with contagious diseases. With the help of the device, it offers an added precautionary measure to ensure adequate temperature monitoring for all patients in the normal ward are done with minimal effort. During the tests on the three participants in different days, we observed that the P1 and P2 had a higher temperature than P3 in particular on the Day 3. That might be due to some "exhaustion" after 2 days. We did suspect the temperature sensor problem but when we calibrated the temperature against the thermometer, it was fine. The reason for no trend in the temperature reason (such as having the circadian rhythm [18]) is due to the participants was actually quite awake (for example, they were chatting and playing with their smart phones) and they did not really fall into sleep.

\section{Conclusions}

This paper presents a web-based MiWi wireless remote temperature-monitoring device using temperature sensors for the axillary measurement. The temperature data were sent via the MiWi wireless protocol to the nurse's station connected to internet. This approach was facilitated by a patient and coordinator set design that connects the process of monitoring, transmitting, alerting and recording of the temperature. The experimental tests on the proposed device indicated that it provides long-distance coverage, a temperature resolution of 0.3 to $0.5^{\circ} \mathrm{C}$ and small standard deviation of approximately $0.3^{\circ} \mathrm{C}$. Importantly, the system will likely to improve the quality of care through 24-hour continuous monitoring of the temperature of patients and reducing the workload of the nursing staff in normal ward.
The remote temperature monitoring device will be further developed into a low-cost product with plug-andplay feature that enable untrained nurse or doctors to use with minimal assistance. The software interface for users will be improved and made easy to be installed and downloaded. For future work involving remote monitoring, the use of different wireless network protocols, such as ZigBee and WiFi, will be compared. A standard medical temperature probe, such as a tympanic probe, and standard disposable tape will be used for final implementation in the hospital. Further evaluation of the proposed system against current measurement techniques needs to be performed. The privacy and security protection and export to clinical information system (CIS) will be studied.

\section{Competing interests}

The authors declare that they have no competing interests.

\section{Authors' contributions}

CSC carried out the design concepts of the wireless remote temperaturemonitoring system and testing. WA carried out the systems integration, programming, and testing on participants. WLW carried out the data analysis. EM provided advice on future works and proof-reading of paper. All authors read and approved the final manuscript.

\section{Acknowledgements}

The authors would like to thanks Newcastle University (UK) and University of Newcastle (Australia) for providing the support during the project.

\section{Author details}

${ }^{1}$ Faculty of Science Agriculture and Engineering, University of Newcastle upon Tyne, Newcastle upon Tyne NE1 7RU, United Kingdom. ${ }^{2}$ Faculty of Engineering and Built Environment, University of Newcastle, Callaghan, NSW 2308, Australia.

Received: 30 September 2014 Accepted: 2 January 2015

Published online: 11 February 2015

\section{References}

1. Rush M, Wetherall A (2003) Temperature measurement: practice guidelines. Paediatr Nurs 15(9):25-28

2. Mahoney C, Odom J (1999) Monitoring intraoperative normothermia: a meta-analysis of outcomes with costs. AANA J 67(2):155-164

3. Yamakoshi KI (2011) In the spotlight: bioinstrumentation. IEEE Rev Biomed Eng 4:6-8

4. Jiann SS, Chun YD, Yeong RW, Wei ZS (2007) A novel fuzzy pain demand index derived from patient-controlled analgesia for postoperative pain. IEEE Trans Biomed Eng 54(12):2123-2132

5. Graichen F, Arnold R, Rohlmann A, Bergmann G (2007) Implantable 9-channel telemetry system for in vivo load measurements with orthopedic implants. IEEE Trans Biomed Eng 54(2):253-261

6. Yang D, Converse MC, Mahvi DM, Webster JG (2007) Measurement and analysis of tissue temperature during microwave liver ablation. IEEE Trans Biomed Eng 54(1):150-155

7. Liu DL, Ebbini ES (2010) Real-time 2-D temperature imaging using ultrasound. IEEE Trans Biomed Eng 57(1):12-16

8. $\mathrm{Ng}$ EY (2005). Is thermal scanner losing its bite in mass screening of fever due to SARS? Medical Physics 32(1):93-7.

9. Bae J, Song K, Lee HW, Cho HW, Yoo HJ (2012) A low-energy crystal-less double-FSK sensor node transceiver for wireless body-area network. IEEE J Solid-State Circuits 47(11):2678-2692

10. Scanail I, Cliodhna N, Ahearne B, Lyons GM (2006) Long-term telemonitoring of mobility trends of elderly people using SMS messaging. IEEE Trans Inf Technol Biomed 10(2):412-413 
11. Malhi K, Mukhopadhyay SC, Schnepper J, Haefke M, Ewald H (2012) A zigbee-based wearable physiological parameters monitoring system. IEEE Journal of Sensors 12(3): 423-430

12. Shahriyar R, Bari MF, Kundu G, Ahamed SI, Akbar MM (2009) Intelligent mobile health monitoring system. Int J Control Automation 2(3):13-27

13. Ivy AC (1945) Comment: what is normal body temperature? Gastroenterology 5:326-329

14. Jeffrey RS (2007) Recovery from severe hyperthermia $\left(45^{\circ} \mathrm{C}\right)$ and rhabdomyolysis induced by methamphetamine body-stuffing. J Emerg Med 8(3):93-95

15. Levander S, Forsberg MC, Wahren LK (2002) Normal oral, rectal, tympanic and axillary body temperature in adult men and women: a systematic literature review. Scand J Caring Sci 16(2):122-128

16. Bridges $E$, Thomas K (2009) Noninvasive measurement of body temperature in critically III patients. Crit Care Nurse 29(3):94-97

17. Langham GE, Maheshwari A, Contrera K, You J, Mascha E, Sessler DI (2009) Noninvasive temperature monitoring in postanesthesia care units. Anesthesiology 111(1):90-96

18. Baker FC, Waner JI, Vieira EF, Taylor SR, Driver HF, Mitchell D (2001) Sleep and 24 hour body temperatures: a comparison in young men, naturally cycling women and women taking hormonal contraceptives. J Physiol 530(3):565-574

Submit your manuscript to a SpringerOpen ${ }^{\circ}$ journal and benefit from:

- Convenient online submission

- Rigorous peer review

- Immediate publication on acceptance

- Open access: articles freely available online

- High visibility within the field

- Retaining the copyright to your article 